\section{Commentary: A pediatric perspective on the Ozaki procedure}

Tara Karamlou, MD, MSc,

Gosta Pettersson, MD, PhD, ${ }^{\mathrm{a}}$ and John J. Nigro, $\mathrm{MD}^{\mathrm{b}}$

Baird and colleagues ${ }^{1}$ describe the first comprehensive application of the Ozaki procedure to a pediatric population. Congenital aortic valve disease is present in up to $2 \%$ of the population ${ }^{2}$ and is a lifelong problem with no discrete cure available. For this reason, management strategies have to be designed in the context of a long life, and there are an increasing number of surgical and interventional options that can be applied to obtain long-term, high-quality palliation. We agree that the Ozaki procedure fits within this paradigm as a surgical technique to help manage congenital aortic valve disease. We also believe that this procedure represents an aortic valve replacement (not a repair or a cure) and likely has a limit to durability that will necessitate subsequent intervention.

The Ozaki procedure was designed and developed for adult patients who require primary aortic valve replacement. Ozaki described the procedure in great detail elsewhere ${ }^{3}$ and he has only made 2 important modifications: The orientation of the pericardial leaflets was changed from having the smooth side (visceral) facing the aorta to having the smooth side facing the ventricle to reduce postoperative platelet consumption, and the leaflets were changed from heterogeneous sizes to equal sizes by altering the commissures to reduce the risk of endocarditis.

Ozaki has not promoted this procedure for children, and Baird and colleagues ${ }^{1}$ are the first to report the adaption of this technique to a pediatric population. This experience suggests that pediatric application of this procedure

\footnotetext{
From the a Division of Congenital Cardiac Surgery, Cleveland Clinic Heart, Vascular, and Thoracic Institute, Cleveland Clinic, Cleveland, Ohio; and ${ }^{\mathrm{b}}$ Division of Cardiothoracic Surgery, Rady Children's Hospital San Diego, University of California San Diego, San Diego, Calif.

Disclosure: Authors have nothing to disclose with regard to commercial support.

Received for publication Feb 27, 2020; revisions received Feb 27, 2020; accepted for publication Feb 27, 2020; available ahead of print March 19, 2020.

Address for reprints: John J. Nigro, MD, Division of Cardiothoracic Surgery, Rady Children's Hospital San Diego, MC 5078, 3020 Children's Way, San Diego, CA 92123 (E-mail: jnigro@ rchsd.org).

J Thorac Cardiovasc Surg 2021;161:1582-3

$0022-5223 / \$ 36.00$

Copyright $($ C 2020 by The American Association for Thoracic Surgery

https://doi.org/10.1016/j.jtcvs.2020.02.128
}

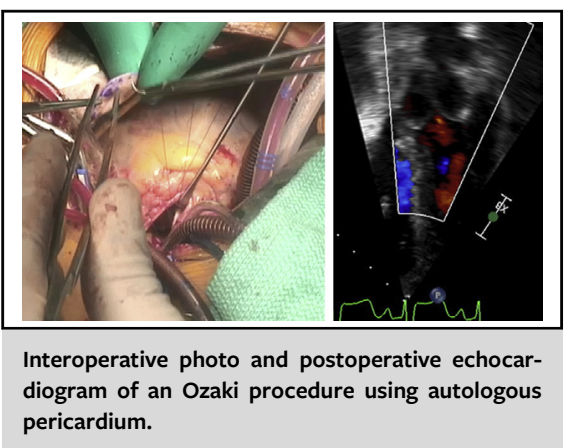

CENTRAL MESSAGE

The Ozaki technique offers another treatment strategy for children and young adults who require aortic valve intervention, but long-term follow-up and context are needed to optimize the benefits.

requires significant alteration. Although not completely elucidated by the authors, several important technical adaptions and modifications of the procedure are described. These adaptions include the need for annulus and root size adjustment (enlargement or reduction), with annular enlargement in 8 patients and aortic sinus enlargement in 20 patients, thus requiring additional harmonization of root dimensions and leaflet size. The duration of the glutaraldehyde fixation of autologous pericardium was also reduced from 10 minutes to 2 minutes to provide more pliable and elastic tissue for leaflet construction. The surface orientation of the pericardium for leaflet construction was adjusted to the opposite orientation relative to that currently proposed by Ozaki, ${ }^{3}$ so that the smooth surface (visceral) faces the aorta due to concerns of leaflet thrombosis and coronary obstruction in smaller roots. Finally, bovine pericardium was used for leaflet construction in patients who conceivably lacked autologous pericardium.

Overall, the procedure described by Baird and colleagues ${ }^{1}$ appears to be effective in the short term and has a low mortality of $3.5 \%$ ( 2 out of 57 patients). Strengths of the study include cohort size $(\mathrm{N}=57)$, echocardiographic follow-up for the entire cohort, and description of the technical adaptions (detailed above) required for pediatric application of this procedure. 
The main limitation of this study is the short follow-up. Echocardiographic surveillance is complete as mentioned, but limited to 1 year in half of patients and 2 years in all patients. Because of this limitation, the utility of this procedure for children beyond 2 years remains unknown. It will be crucial to obtain longer-term data to determine the durability of this procedure for children who notoriously experience early tissue valve failure and somatic outgrowth. Variability in this series was not isolated to technique because the procedure was applied to a wide range of patient ages and sizes and a mixture of underlying pathophysiology of valve failure (24 patients had aortic insufficiency, 27 patients had combined aortic stenosis and insufficiency, and 6 patients had aortic stenosis). This diversity may vicariously reflect the versatility of the procedure; but technical modifications may influence valve performance and durability and the heterogeneous cohort may introduce confounding factors that may all complicate evaluation of the utility of the Ozaki procedure for children. It is concerning that 12 patients in this study were found to have decreased leaflet mobility and that since November 2017 the authors have utilized warfarin therapy. The underlying process will require elucidation and dedicated imaging analogous to the transcatheter aortic valve implant surveillance paradigm for hypoattenuating leaflet thickening ${ }^{4}$ may be warranted. Although growth is desirable for this population, the progressive annular enlargement in this series during short follow-up is worrisome. The authors have ascribed this dilation to somatic growth, but continued enlargement of the annulus would conceivably lead to valve dysfunction because the reconstructed leaflets are not expected to grow with the annulus. It will be very important to determine the long-term behavior of bovine pericardium used in this series.

This study suggests that the Ozaki procedure can be adapted to and utilized in pediatric populations, but the use of this procedure relative to alternatives is difficult to interpret because the other options for these patients were not described. Due to the chronic nature of aortic valve disease and limitations for all the available therapies, we must determine how to optimize both timing and triage to a particular valve intervention for the individual child. The Ross procedure remains a very attractive option in patients with a normal pulmonary valve, who require growth, and who have a small aortic annulus. ${ }^{5}$ A mechanical valve replacement remains a reasonable first choice for older children and adults with bicuspid aortic valves with thickened and restricted leaflets that are considered unrepairable and who are able and willing to take warfarin. For children between these extremes there is a role for the Ozaki procedure because even with bovine pericardium it may be more predictable than repair with leaflet extensions. Additionally, the Ozaki procedure may be an attractive choice in developing countries challenged by the cost and availability of valve prostheses.

The authors are to be congratulated for this large experience, their clinical results, and the adaption of the Ozaki procedure to a pediatric population. We are very cautious regarding the interpretation of these results until longer follow-up is available. Because of the limited follow-up, the true utility of this procedure and how it fits into the armamentarium for care of children with aortic valve disease remains unknown. The art of congenital aortic disease management will likely require mastery of many techniques, an understanding of how and when to deploy these techniques, and the sequence in which they should be applied. All of this underscores the need for long-term follow-up data that include functional capacity and quality of life parameters, and the evolution of focused initiatives to ensure good long-term and high-quality care for patients with congenital aortic valve disease.

\section{References}

1. Baird C, Sefton B, Chavez M, Sleeper LA, Marx GR, del Nido PJ. Congenital aortic and truncal valve reconstruction using the Ozaki technique: short-term clinical results. J Thorac Cardiovasc Surg. 2021;161:1567-77.

2. Hoffman JI, Kaplan S. The incidence of congenital heart disease. J Am Coll Car diol. 2002;39:1890-900.

3. Ozaki S, Kawase I, Yamashita H, Nozawa Y, Takatoh M, Hagiwara S, et al. Aortic valve reconstruction using autologous pericardium for patients aged less than 60 years. J Thorac Cardiovasc Surg. 2014;148:934-8.

4. Polzin A, Veulemans V, Dannenberg L, Kelm M, Zeus T. HALT in TAVR: what about aspirin? JACC Cardiovasc Interv. 2019;12:894.

5. Bansal N, Kumar SR, Baker CJ, Lemus R, Wells WJ, Starnes VA. Age-related outcomes of the Ross procedure over 20 years. Ann Thorac Surg. 2015;99:2077-83. 